\title{
Relationship between sarcopenia and physical activity in older people: a systematic review and meta-analysis
}

\author{
Michal Steffl' \\ Richard W Bohannon ${ }^{2}$ \\ Lenka Sontakova' \\ James J Tufano' \\ Kate Shiells ${ }^{3}$ \\ Iva Holmerova ${ }^{3}$ \\ 'Department of Physiology and \\ Biochemistry, Faculty of Physical \\ Education and Sport, Charles \\ University Prague, Prague, Czech \\ Republic; ${ }^{2}$ Department of Physical \\ Therapy, College of Pharmacy and \\ Health Sciences, Campbell University, \\ Buies Creek, NC, USA; ${ }^{3}$ Faculty of \\ Humanities, Centre of Gerontology, \\ Charles University Prague, Prague, \\ Czech Republic
}

This article was published in the following Dove Press journal:

Clinical Interventions in Aging

17 May 2017

Number of times this article has been viewed

\begin{abstract}
Physical activity (PA) has been identified as beneficial for many diseases and health disorders, including sarcopenia. The positive influence of PA interventions on sarcopenia has been described previously on many occasions. Current reviews on the topic include studies with varied PA interventions for sarcopenia; nevertheless, no systematic review exploring the effects of PA in general on sarcopenia has been published. The main aim of this study was to explore the relationship between PA and sarcopenia in older people on the basis of cross-sectional and cohort studies. We searched PubMed, Scopus, EBSCOhost, and ScienceDirect for articles addressing the relationship between PA and sarcopenia. Twenty-five articles were ultimately included in the qualitative and quantitative syntheses. A statistically significant association between PA and sarcopenia was documented in most of the studies, as well as the protective role of PA against sarcopenia development. Furthermore, the meta-analysis indicated that PA reduces the odds of acquiring sarcopenia in later life (odds ratio $[\mathrm{OR}]=0.45 ; 95 \%$ confidence interval $[\mathrm{CI}] 0.37-0.55)$. The results of this systematic review and meta-analysis confirm the beneficial influence of PA in general for the prevention of sarcopenia.
\end{abstract}

Keywords: aging, sarcopenia, physical activity

\section{Introduction}

Although diseases related to the aging process are problematic themselves, they rarely occur in isolation and the effects of one may spark the onset of another. As such ailments progress, the importance of physical activity (PA) remains high, with previous research confirming that regular PA is essential for healthy aging. ${ }^{1}$ Specifically, PA plays a substantial role in lowering the risk of coronary heart disease, ${ }^{2}$ obesity, ${ }^{3}$ type 2 diabetes, ${ }^{4}$ hypertension, ${ }^{5}$ peripheral vascular disease,${ }^{6}$ high cholesterol, ${ }^{7}$ osteoporosis, ${ }^{8}$ osteoarthritis, ${ }^{9}$ and chronic obstructive pulmonary disease. ${ }^{10}$ Although PA may have an indirect impact on some health aspects, it has a direct impact on muscle quality and quantity. ${ }^{11}$

Sarcopenia, which was first described by Rosenberg in $1989^{12}$ as the progressive decrease in muscle mass and strength during aging, is a syndrome that is directly affected by PA. ${ }^{13-15}$ Soon after sarcopenia was defined, muscle mass assessment had been recommended as the main sarcopenia diagnosing method. Baumgartner et $\mathrm{al}^{16}$ proposed that the appendicular skeletal muscle mass index (ASMMI) should be the main indicator, and the cutoff point was established as two standard deviations below the mean of a young reference group. Even though this measurement is always expressed in relative terms (muscle mass in kilograms divided by body
Correspondence: Michal Steffl Department of Physiology and Biochemistry, Faculty of Physical Education and Sport, Charles University, José Martího 3I, Prague 6, 162 52, Czech Republic

Email steffl@ftvs.cuni.cz 
height in meter squared, resulting in $\mathrm{kg} / \mathrm{m}^{2}$ ), many different names have been suggested, eg, appendicular lean mass index (ALMI), fat-free mass index (FFMI), relative skeletal muscle mass index (RSMI), and muscle mass index (MMI), and occasionally skeletal muscle mass (SMM) alone serves as an indicator of sarcopenia. Computed tomography (CT) and magnetic resonance imaging (MRI) are gold standards for measuring muscle mass in research. The dual-energy X-ray absorptiometry (DXA) is the preferred alternative method for research and clinical use; ${ }^{17}$ however, bioelectrical impedance analysis (BIA) has been found as a relevant alternative. ${ }^{18}$ Except these methods, the mid-upper arm muscle circumference (MAMC) has been proposed as an alternative tool for muscle mass estimation. ${ }^{19}$ Later, several groups were formed for sarcopenia consensus on definition and diagnosis in Europe - the European Working Group on Sarcopenia in Older People (EWGSOP), ${ }^{17}$ in Asia - the Asian Working Group for Sarcopenia (AWGS), ${ }^{20}$ and except them the International Working Group on Sarcopenia (IWGS). ${ }^{21}$ These groups recommended including muscle strength and physical performance measurement as the additional methods for sarcopenia diagnosing. Currently, the EWGSOP algorithm is the most widely used method in research and in clinical practice.

Previous research has shown that physical inactivity contributes to the development of sarcopenia, ${ }^{22,23}$ and other studies have shown that PA increases muscle strength ${ }^{24,25}$ and muscle mass ${ }^{26,27}$ in older adults. Therefore, a strong link has emerged between PA and a lower prevalence of sarcopenia. ${ }^{28-31}$ Specifically, resistance training is generally considered to be the best countermeasure for preventing sarcopenia. ${ }^{11,32-38}$

Although many reviews and meta-analyses have summarized the effects of individual or combined interventions (eg, resistance training and nutritional supplementation) on sarcopenia, a systematic review and meta-analysis of the effects of PA defined as general activity that requires more energy than resting metabolic rate (eg, exercising, strengthening, walking, working in the garden, and so on) on sarcopenia has not been published. Therefore, the main aim of this systematic review and meta-analysis was to describe the relationship between PA and the presence of sarcopenia.

\section{Methods}

This systematic review and meta-analysis, in accordance with the recommendations and criteria as outlined in the Preferred Reporting Items for Systematic Reviews and
Meta-Analyses (PRISMA) statement, ${ }^{39}$ focused on crosssectional and cohort studies.

\section{Criteria for considering studies for this study}

To be included in the analyses, studies had to meet the following conditions: only data from cross-sectional studies and follow-up or baseline datasets of longitudinal cohort studies were included, date of publication 1989-2017, English language, and participants have to be older than 40 years. As PA, there were considered every activity requiring increased energy output without regard of frequency and intensity, sarcopenia has to be diagnosed by some of the standard recommendation. There had to be data presented from regression models, which included PA as the independent variable and sarcopenia as the dependent variable, and odds ratio (OR) had to be used as the effect size in those regression models for the systematic review. For the meta-analysis, the raw data reporting numerically PA habits by both sarcopenic and nonsarcopenic individuals had to be presented.

\section{Search methods for identification of studies}

Appropriate articles were manually identified through searches using four electronic databases: PubMed, Scopus, EBSCOhost, and ScienceDirect and through the reference lists of publications identified in this search. The search stream that was used in all the databases is presented in Table 1. This process was conducted by the first and third authors; the searches were done by the first and last authors independently. There was no disagreement between those coauthors during the process. The articles were collected and sorted using the software EndNote X5 for managing bibliographies.

\section{Data collection and analysis}

All abstracts were explored by the first and third authors independently with the aim of identifying relevant articles. During the first step of this process, duplicate articles and reviews were removed, then full texts of the remaining articles were systematically examined for inclusion or exclusion, and the articles lacking the required information about PA and sarcopenia were removed. Subsequently, the remaining articles were included in the synthesis. Additionally, the aforementioned protocol was completed on relevant articles that were identified within the reference lists of the articles identified through database searching. 
Table I Search strategies used with four databases to identify articles describing the relationship between sarcopenia and physical activities

\begin{tabular}{lll}
\hline $\begin{array}{l}\text { Database } \\
\text { (yield) }\end{array}$ & Search terms & $\begin{array}{l}\text { Record identified } \\
\text { through searching }\end{array}$ \\
\hline PubMed & $\begin{array}{l}\text { Search (sarcopenia[Title]) AND (“physical activit*”) AND } \\
\text { (cross-sectional OR cohort) }\end{array}$ & 85 \\
Scopus & (TITLE (sarcopenia) AND TITLE-ABS-KEY (“physical \\
& activit*”) AND TITLE-ABS-KEY (cross-sectional OR cohort)) & 54 \\
EBSCOhost & TI sarcopenia AND TX “physical activit”” AND TX (cross- & 182 \\
ScienceDirect & sectional OR cohort) & 22 \\
\hline
\end{tabular}

Note: January 4, 2017 - record identified through database searching: 343.

After collection of the relevant articles, the NewcastleOttawa Scale (NOS) was used to assess the quality of nonrandomized studies in meta-analyses to eliminate the risk of bias. ${ }^{40}$ This was carried out independently by the fourth and fifth authors. There was no disagreement between them during the process.

\section{Measures of effect sizes}

The Cochran-Mantel-Haenszel statistical method ${ }^{41}$ based on a fixed-effect model was used to calculate an effect size of PA on sarcopenia in the meta-analysis. The OR was calculated as the effect size of PA on sarcopenia. In this article, the OR estimated the odds of demonstrating sarcopenia while accounting for PA. An OR less than 1 favors PA, indicating that PA decreases the risk (odds) of sarcopenia, and an OR greater than 1 suggests that PA increases the risk (odds) of sarcopenia. A sensitivity analysis was carried out to reach the best estimation. During the sensitivity analysis, those studies that might have had an influence on heterogeneity because of publication bias were removed. Through the sensitivity analysis, the best OR estimation represented by the highest value of a test for the overall effect $(Z)$ taking into account heterogeneity and publication bias was calculated. An index $I^{2}$, which does not depend upon the number of studies, the type of outcome data, or the choice of treatment effect, was used to quantify the impact of heterogeneity and to assess inconsistency. $I^{2}$ can be readily calculated from basic results obtained from a typical meta-analysis as $I^{2}=100 \% \times(\mathrm{Q}-d f) / \mathrm{Q}$, where $\mathrm{Q}$ is standard Cochran's heterogeneity statistic and $\mathrm{df}$ the degrees of freedom. A rough guide to interpretation of $I^{2}$ is as follows: 0 to $40 \%$ might not be important, $30 \%$ to $60 \%$ may represent moderate heterogeneity, $50 \%$ to $90 \%$ may represent substantial heterogeneity, and $75 \%$ to $100 \%$ represents considerable heterogeneity. ${ }^{42}$
Funnel plots were used for visualizing biases ${ }^{43} \mathrm{~A}$ funnel plot is a simple scatter plot of exposing the effect estimated from individual studies against some measures of each study's size or precision. ${ }^{44}$ Statistics were carried out using Review Manager 5.3.

\section{Results}

\section{Description of studies and study population}

Figure 1 summarizes the yield of the search process. Of 354 articles identified as potentially relevant by the database searching, 19 were included. An additional six articles identified through article reference lists were added. Excluded articles are presented in Table S1. Ultimately, 25 total studies were included in the qualitative and quantitative syntheses, comprised of 17 cross-sectional ${ }^{30,31,45-59}$ and 8 cohort studies. ${ }^{29,60-66}$ From these 25 articles, 20 were used in the systematic review and 14 were used in the meta-analysis: some articles were used for both, but all 25 articles were used in one way or another. Data from 40,007 individuals (21,222 males and 18,785 females) were obtained from all 25 studies. The mean age of the participants was $71.7 \pm 4.9$ for nonsarcopenic males and $74.9 \pm 5.6$ for sarcopenic males and $73.1 \pm 4.7$ for nonsarcopenic females and $76.1 \pm 5.0$ for sarcopenic females. All participants were over 60 years old apart from subjects within four studies: Beavers et al ( $>40$ years old $),{ }^{45}$ de Castro et al $(55-68$ years old $),{ }^{46}$ Castillo et al (55-98 years old) ${ }^{62}$ and Park et al ( $>50$ years old) ${ }^{55}$ The age ranged from 40 to 106 years. Participants lived in a community in 23 cases, one time in a nursing home,,$^{50}$ and one time participants were recruited from a hospital. ${ }^{53} \mathrm{PA}$ was quantified using several different methods, but the most common was a self-report questionnaire. In most studies, PA was divided into several categories. Sarcopenia diagnostics 


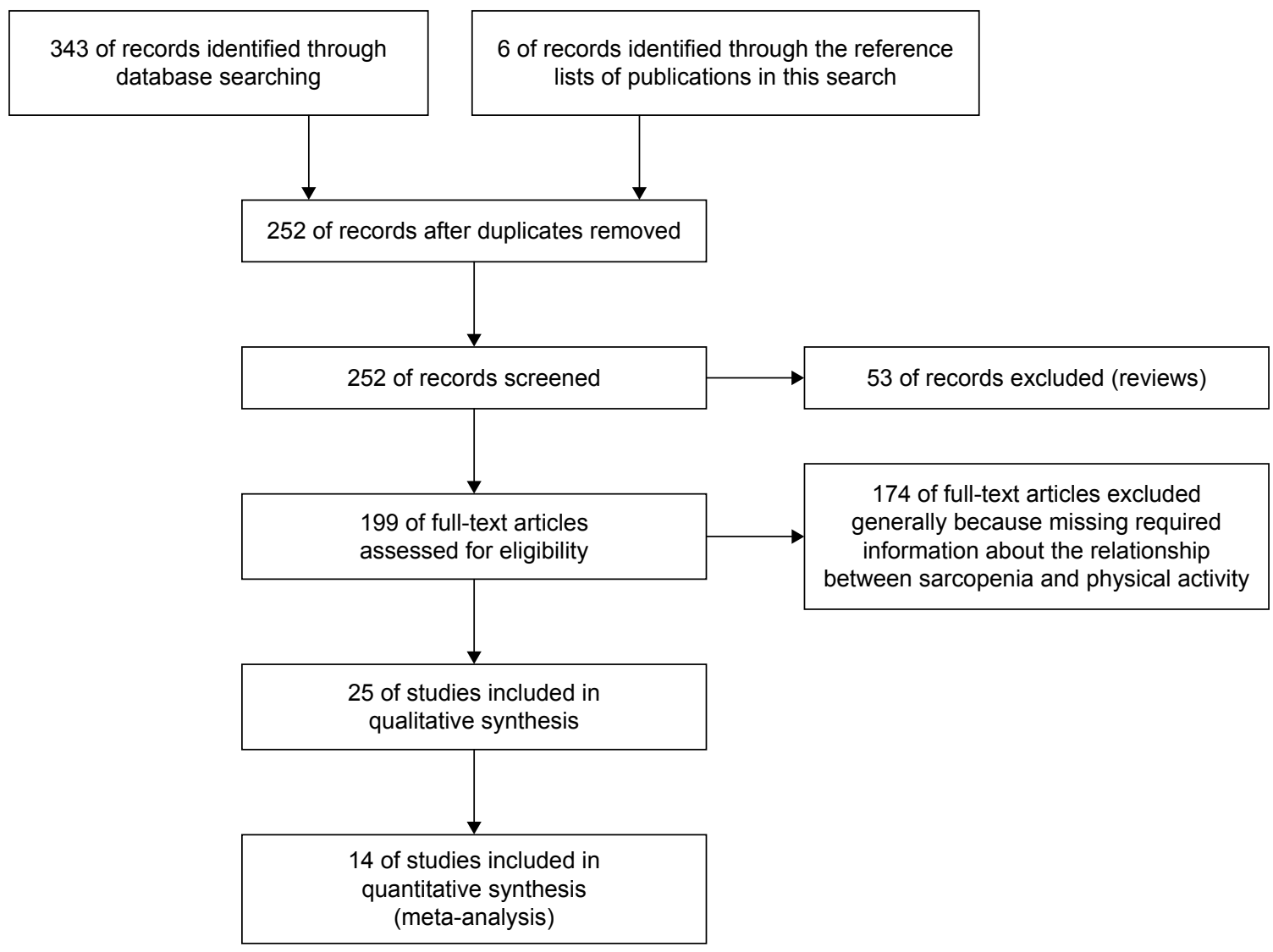

Figure I Flowchart showing how the reviewed articles were identified and selected.

were based on body composition measurements in most cases. The EWGSOP algorithm was used in seven studies, and AWGS criteria and IWGS criteria were each used one time (Table 2). The quality of the included studies was sufficient according to the NOS score, and no study was excluded due to that analysis (Table S2).

\section{Systematic review}

Most of the regression models suggested that PA might help preserve muscle mass because only four of 32 ORs were above 1 , and only one study ${ }^{49}$ estimated PA to be a significant risk factor for sarcopenia in females. However, 13 of 32 regression models estimated PA to be a significant protective factor against sarcopenia in older people (Table 3). Additionally, six of nine regression models estimated that physical inactivity was a significant risk factor for sarcopenia in older people (Table 4).

\section{Meta-analysis}

In all the analyses, the article by Goodman et $\mathrm{al}^{49}$ stand out of the funnel plot that signalized the increased risk of bias and for the same reason the article by Park et al ${ }^{55}$ was excluded during sensitivity analysis in the analysis males and females together.

For males, data from eight studies were initially included in the first analysis, with one study ${ }^{49}$ later excluded due to publication bias, thereby resulting in acceptable heterogeneity, from $I^{2}=73 \%$ to $I^{2}=4 \%$. After the exclusion of this study, the OR $(95 \%$ confidence interval [CI]) for males $(n=3,881)$ was $0.46(0.37-0.58)$, Cochran $\mathrm{Q}=5.2, d f=5(P=0.390)$, indicating that $P A$ reduced the odds of males suffering from sarcopenia. The test for overall effect was quite strong $Z=6.50$, which was statistically significant $(P<0.00001)$. The forest plot is shown in Figure 2.

For females, data from seven studies were included in the first analysis, but data from Goodman et a ${ }^{49}$ were excluded for the same reason, reducing heterogeneity from $I^{2}=75 \%$ to an acceptable $I^{2}=29 \%$. After the exclusion of this study, the OR $(95 \% \mathrm{CI})$ for females $(n=6,234)$ was $0.65(0.52-0.81)$, Cochran $\mathrm{Q}=7.8, d f=5(P=0.290)$ indicating that $\mathrm{PA}$ reduced the odds of females demonstrating sarcopenia. The test for overall effect was weaker than in males at $Z=3.79$; however, it was still statistically significant $(P<0.0001)$. The forest plot is shown in Figure 3. 
Table 2 Summary of studies describing the classification of PA and sarcopenia diagnostics

\begin{tabular}{|c|c|c|}
\hline \multirow[t]{2}{*}{ Study } & \multirow[t]{2}{*}{ Design $^{a}$} & Classification of PA \\
\hline & & Sarcopenia diagnostics \\
\hline \multirow[t]{2}{*}{ Aggio et $a^{160}$} & Cohort ${ }^{\mathrm{b}}$ & Physical monitoring: accelerometry \\
\hline & & $\begin{array}{l}\text { EWGSOP algorithm, }{ }^{17} \text { SMM }(\mathrm{kg}) \text { by MAMC, }{ }^{72} \text { the lowest two-fifths of the MAMC } \\
\text { distribution }\end{array}$ \\
\hline \multirow[t]{3}{*}{ Akune et $\mathrm{a}^{29}$} & Cohort $^{\mathrm{b}}$ & Survey: categorization of past PA based on yes/no \\
\hline & & Survey: categorization of past current walking habit based on yes/no \\
\hline & & $\begin{array}{l}\text { EWGSOP algorithm, }{ }^{17} \mathrm{SMI}\left(\mathrm{kg} / \mathrm{m}^{2}\right) \text { by BIA; cutoff } 7.0 \mathrm{~kg} / \mathrm{m}^{2} \text { for males and } 5.8 \mathrm{~kg} / \mathrm{m}^{2} \text { for } \\
\text { females }\end{array}$ \\
\hline \multirow[t]{2}{*}{ Atkins et $a^{61}$} & Cohort ${ }^{\mathrm{b}}$ & $\begin{array}{l}\text { Survey: current PA classified as: inactive, occasional, light, moderate, moderately vigorous, } \\
\text { vigorous }\end{array}$ \\
\hline & & FFMI $\left(\mathrm{kg} / \mathrm{m}^{2}\right)$ by BIA, $<$ Ist quartile of the distribution of FFMI; cutoff $15.96 \mathrm{~kg} / \mathrm{m}^{2}$ \\
\hline \multirow[t]{2}{*}{ Beavers et a $\left.\right|^{45}$} & Cross-sectional & $\begin{array}{l}\text { Survey: current PA classified as: inactive }<4 \text {, low } 4-1 \mid \text {, moderate } 12-20 \text {, high }>20 \text { times } \\
\text { per month }\end{array}$ \\
\hline & & $\begin{array}{l}\text { SMM }(\mathrm{kg}) \text { by BIA, sarcopenia class I and II of }<I \text { SD, respectively, }<2 \text { SD of young reference } \\
\text { group from this study }\end{array}$ \\
\hline \multirow[t]{2}{*}{ Castillo et a ${ }^{62}$} & Cohort ${ }^{\mathrm{b}}$ & Survey: regular PA three or more times per week - yes/no \\
\hline & & FFM $(\mathrm{kg})$ by BIA, $<2$ SD of a young reference group from Pichard et al study ${ }^{73}$ \\
\hline de Castro & Cross-sectional & International Physical Activity Questionnaire (IPAQ) - long version ${ }^{74}$ \\
\hline et $\mathrm{al}^{46}$ & & $\begin{array}{l}\text { ALMI }\left(\mathrm{kg} / \mathrm{m}^{2}\right) \text { by DXA, }<\text { I SD of a young reference group from this study, cutoff } 7.3 \mathrm{~kg} / \mathrm{m}^{2} \\
\text { for females }\end{array}$ \\
\hline \multirow[t]{2}{*}{ da Silva et al ${ }^{47}$} & Cross-sectional & Survey: categorization of past PA based on yes/no \\
\hline & & SMI $\left(\mathrm{kg} / \mathrm{m}^{2}\right)$ by BIA, cutoff $16.7 \mathrm{~kg} / \mathrm{m}^{2}$ adopted from Atkins et al study ${ }^{75}$ \\
\hline \multirow{3}{*}{$\begin{array}{l}\text { Domiciano } \\
\text { et } \mathrm{al}^{63}\end{array}$} & Cohort $^{\mathrm{b}}$ & An interviewer-mediated standardized questionnaire adapted from National Health \\
\hline & & Interview Survey Basic Questionnaire ${ }^{76}$ \\
\hline & & RSMI $\left(\mathrm{kg} / \mathrm{m}^{2}\right)$ by DXA, cutoff $5.45 \mathrm{~kg} / \mathrm{m}^{2}$ for females adopted from Baumgartner et al study 16 \\
\hline \multirow[t]{2}{*}{ Dutra et a $\left.\right|^{48}$} & Cross-sectional & International Physical Activity Questionnaire (IPAQ) - long version ${ }^{74,77}$ \\
\hline & & $\begin{array}{l}\text { EWGSOP algorithm, SMI }\left(\mathrm{kg} / \mathrm{m}^{2}\right) \text { by the Lee equation, }{ }^{78} \text { cutoff } 6.75 \mathrm{~kg} / \mathrm{m}^{2} \text { for females } \\
\text { adopted from Janssen et al study }\end{array}$ \\
\hline \multirow[t]{3}{*}{ Figueiredo et al ${ }^{64}$} & Cohort ${ }^{\mathrm{b}}$ & An interviewer-mediated standardized questionnaire adapted from National Health \\
\hline & & Interview Survey Basic Questionnaire ${ }^{76}$ \\
\hline & & ASMMI $\left(\mathrm{kg} / \mathrm{m}^{2}\right)$ by DXA, cutoff $7.26 \mathrm{~kg} / \mathrm{m}^{2}$ for males adopted from Baumgartner et al study 16 \\
\hline \multirow[t]{2}{*}{ Goodman et al ${ }^{49}$} & Cross-sectional & Survey: average level of PA each day classified as: low, moderate, heavy \\
\hline & & SMI $\left(\mathrm{kg} / \mathrm{m}^{2}\right)$ by DXA, sarcopenia class I $<$ ISD of a young reference group from this study \\
\hline \multirow[t]{3}{*}{ Kim et $\mathrm{al}^{30}$} & Cross-sectional & International Physical Activity Questionnaire (IPAQ) - long version ${ }^{77}$ \\
\hline & & ASMMI $\left(\mathrm{kg} / \mathrm{m}^{2}\right)$ by DXA, $<2 \mathrm{SD}$ of a young reference group from this study, cutoff \\
\hline & & $6.52 \mathrm{~kg} / \mathrm{m}^{2}$ for males \\
\hline \multirow[t]{2}{*}{ Landi et al ${ }^{50}$} & Cross-sectional & Minimum Data Set assessment form for the Nursing Home (MDS-NH) ${ }^{80,81}$ \\
\hline & & $\begin{array}{l}\text { EWGSOP algorithm, SMI }\left(\mathrm{kg} / \mathrm{m}^{2}\right) \text { by BIA, cutoff } 8.87 \mathrm{~kg} / \mathrm{m}^{2} \text { for males and } 6.42 \mathrm{~kg} / \mathrm{m}^{2} \text { for } \\
\text { females adopted from NHANES III }\end{array}$ \\
\hline \multirow[t]{2}{*}{ Lau et $\mathrm{a}^{51}$} & Cross-sectional & Survey: categorization of load-bearing exercise based on yes/no \\
\hline & & ASMMI $\left(\mathrm{kg} / \mathrm{m}^{2}\right)$ by DXA, $<2.0 \mathrm{SD}$ of a young reference group from this study \\
\hline \multirow[t]{2}{*}{ Lin et $\mathrm{al}^{52}$} & Cross-sectional & Survey: categorization of regular exercise habits based on yes/no \\
\hline & & $\begin{array}{l}\text { EWGSOP algorithm, SMI }\left(\mathrm{kg} / \mathrm{m}^{2}\right) \text { by DXA, }<2 \mathrm{SD} \text { of a young reference group from } \\
\text { this study }\end{array}$ \\
\hline \multirow[t]{2}{*}{ Martinez et $\mathrm{a}^{53}$} & Cross-sectional & Survey: categorization of PA prior admission less than $2 \times$ per week based on yes/no \\
\hline & & MMI $\left(\mathrm{kg} / \mathrm{m}^{2}\right)$ by the Lee equation, ${ }^{78} \leq 20$ th percentile, cutoff $8.90 \mathrm{~kg} / \mathrm{m}^{2}$ for males and \\
\hline \multirow{3}{*}{ Murphy et a $\left.\right|^{54}$} & Cross-sectional & Survey: PA was assessed according to $\mathrm{kcal} / \mathrm{wk}$ spent by exercising in the prior week \\
\hline & & as: $<500,500-1,499,>1,500 \mathrm{kcal} / \mathrm{wk}$ \\
\hline & & ALMI $\left(\mathrm{kg} / \mathrm{m}^{2}\right)$ by DXA, $\leq 20$ th percentile, cutoff $7.95 \mathrm{~kg} / \mathrm{m}^{2}$ for males and $6.24 \mathrm{~kg} / \mathrm{m}^{2}$ for females \\
\hline \multirow[t]{2}{*}{ Park et al $\left.\right|^{55}$} & Cross-sectional & International Physical Activity Questionnaire (IPAQ) - long version ${ }^{77}$ \\
\hline & & SMI $\left(\mathrm{kg} / \mathrm{m}^{2}\right)$ by DXA, $<2 \mathrm{SD}$ of a young reference group from this study \\
\hline \multirow[t]{3}{*}{ Rolland et al ${ }^{65}$} & Cohort ${ }^{c}$ & $\begin{array}{l}\text { Survey: categorization of recreational PA for } \geq \mathrm{I} \mathrm{h/wk} \text { for the past month or more based on } \\
\text { yes/no }\end{array}$ \\
\hline & & SMI $\left(\mathrm{kg} / \mathrm{m}^{2}\right)$ by DXA, $<2$ SD of a reference population from the Rosetta Study, ${ }^{82}$ cutoff \\
\hline & & $5.45 \mathrm{~kg} / \mathrm{m}^{2}$ for females \\
\hline
\end{tabular}


Table 2 (Continued)

\begin{tabular}{|c|c|c|}
\hline \multirow[t]{2}{*}{ Study } & \multirow[t]{2}{*}{ Design $^{a}$} & Classification of PA \\
\hline & & Sarcopenia diagnostics \\
\hline \multirow[t]{2}{*}{ Ryu et $\mathrm{al}^{31}$} & Cross-sectional & International Physical Activity Questionnaire (IPAQ) - long version ${ }^{77}$ \\
\hline & & ASMMI $\left(\mathrm{kg} / \mathrm{m}^{2}\right)$ by DXA, $<2$ SD of a young reference group from this study \\
\hline \multirow{3}{*}{$\begin{array}{l}\text { Silva Alexandre } \\
\text { et } \mathrm{a}^{56}\end{array}$} & Cross-sectional & International Physical Activity Questionnaire (IPAQ) - long version ${ }^{74}$ \\
\hline & & EWGSOP algorithm, ${ }^{17} \mathrm{SMI}\left(\mathrm{kg} / \mathrm{m}^{2}\right)$ by the Lee equation, ${ }^{78} \leq 20$ th percentile, cutoff \\
\hline & & $8.90 \mathrm{~kg} / \mathrm{m}^{2}$ for males and $6.37 \mathrm{~kg} / \mathrm{m}^{2}$ for females \\
\hline \multirow{2}{*}{$\begin{array}{l}\text { Tramontano } \\
\text { et al }\left.\right|^{57}\end{array}$} & Cross-sectional & Global Physical Activity Questionnaire (GPAQ) ${ }^{83}$ \\
\hline & & $\begin{array}{l}\text { IWGS criteria, }{ }^{21} \text { ASMMI }\left(\mathrm{kg} / \mathrm{m}^{2}\right) \text { by DXA, cutoff } 7.23 \mathrm{~kg} / \mathrm{m}^{2} \text { for males and } 5.67 \mathrm{~kg} / \mathrm{m}^{2} \text { for } \\
\text { females }\end{array}$ \\
\hline \multirow[t]{2}{*}{ Volpato et al ${ }^{58}$} & Cross-sectional & Survey: PA was divided as: low, moderate/high \\
\hline & & $\begin{array}{l}\text { EWGSOP algorithm, }{ }^{17} \mathrm{SMI}\left(\mathrm{kg} / \mathrm{m}^{2}\right) \text { by BIA, cutoff } 8.87 \mathrm{~kg} / \mathrm{m}^{2} \text { for males and } 6.42 \mathrm{~kg} / \mathrm{m}^{2} \text { for } \\
\text { females according to EWGSOP17 }\end{array}$ \\
\hline \multirow[t]{2}{*}{ Yu et $\mathrm{al}^{66}$} & Cohort $^{\mathrm{d}}$ & Physical Activity Scale of the Elderly (PASE) ${ }^{84}$ \\
\hline & & $\begin{array}{l}\text { EWGSOP algorithm, }{ }^{17} \mathrm{SMI}\left(\mathrm{kg} / \mathrm{m}^{2}\right) \text { by DXA, the lowest quintile, cutoff } 6.52 \mathrm{~kg} / \mathrm{m}^{2} \text { for males } \\
\text { and } 5.44 \mathrm{~kg} / \mathrm{m}^{2} \text { for females }\end{array}$ \\
\hline \multirow[t]{2}{*}{ Zeng et a $1^{59}$} & Cross-sectional & $\begin{array}{l}\text { Survey: engaging in physical exercise at least once a week and lasting for } 30 \text { min or } \\
\text { more - yes/no }\end{array}$ \\
\hline & & AWGS criteria, ${ }^{20} \mathrm{SMI}\left(\mathrm{kg} / \mathrm{m}^{2}\right)$ by BIA \\
\hline
\end{tabular}

Notes: ${ }^{a}$ As stated by the authors. ${ }^{b}$ Follow-up dataset. 'Baseline dataset. ${ }^{\mathrm{d} B a s e l i n e}$ to 2 years.

Abbreviations: PA, physical activity; EWGSOP, European Working Group on Sarcopenia in Older People; SMM, skeletal muscle mass; MAMC, mid-upper arm muscle circumference; SMI, skeletal muscle mass index; BIA, bioelectrical impedance analysis; FFMI, fat-free mass index; SD, standard deviation; ALMI, appendicular lean mass index; DXA, dual-energy X-ray absorptiometry; RSMI, relative skeletal muscle mass index; AWGS, Asian Working Group for Sarcopenia.

When combining males and females (nine studies), and after excluding two studies ${ }^{49,55}$ through sensitivity analysis, data from the overall population $(n=4,605)$ showed the strongest estimation with an OR $(95 \% \mathrm{CI})$ of $0.45(0.37-0.55)$, Cochran $\mathrm{Q}=8.1, d f=6(P=0.230)$ indicating that $\mathrm{PA}$ reduced the odds of patients suffering from sarcopenia. The test for overall effect was strong at $Z=7.76(P<0.00001)$, and heterogeneity was sufficiently acceptable $I^{2}=26 \%$. The forest plot from this analysis is presented in Figure 4.

\section{Discussion}

An emerging body of evidence shows that PA plays a preventive role against many diseases such as coronary heart disease, obesity, type 2 diabetes, hypertension, peripheral vascular disease, high cholesterol, osteoporosis, osteoarthritis, and chronic obstructive pulmonary disease. Data from our systematic review and meta-analysis, similar to that of previous authors, ${ }^{14,15}$ also show that PA protects against sarcopenia. Our results are also in concordance with

Table 3 Relationship between sarcopenia and physical activity, according to multiple regression models

\begin{tabular}{|c|c|c|c|c|c|}
\hline Study & $\mathbf{N}$ & Variable & Status & $\begin{array}{l}\text { Multiple logistic regression models } \\
\text { adjusted for }\end{array}$ & OR $(95 \% \mathrm{Cl})$ \\
\hline \multicolumn{6}{|l|}{ Females } \\
\hline \multirow[t]{2}{*}{ Akune et $\mathrm{al}^{29}$} & 651 & Current walking habits & Yes vs no & Age and BMI & $0.75(0.39-1.44)$ \\
\hline & & Exercise habits in middle age & Yes vs no & Age and BMI & $0.55(0.27-1.13)$ \\
\hline Castillo et al ${ }^{62}$ & 1,006 & Exercise 3+ times/wk & Yes vs no & $\begin{array}{l}\text { Age, alcohol use, and current } \\
\text { smoking status }\end{array}$ & $0.5 \mathrm{I}(0.30-0.89)^{*}$ \\
\hline $\begin{array}{l}\text { de Castro } \\
\text { et } \mathrm{al}^{46}\end{array}$ & 91 & Physical activity level & - & $\begin{array}{l}\text { Age, total cholesterol, LDL, HDL, } \\
\text { diabetes, WHR, WC, Cl, WHER, } \\
\text { and BMI }\end{array}$ & $0.54(0.13-2.27)$ \\
\hline \multirow[t]{2}{*}{$\begin{array}{l}\text { Goodman } \\
\text { et al }{ }^{49}\end{array}$} & 374 & $\begin{array}{l}\text { Average level of physical } \\
\text { activity each day }\end{array}$ & Moderate vs low & Unadjusted & $1.30(1.01-1.75) *$ \\
\hline & & & Heavy vs low & Unadjusted & $1.14(0.40-4.23)$ \\
\hline \multirow[t]{2}{*}{ Ryu et $\mathrm{al}^{31}$} & $\mathrm{I}, 324$ & Physical activity level & Moderate vs low & Age & I.0I (0.65-I.57) \\
\hline & & & High vs low & Age & $0.76(0.45-1.29)$ \\
\hline \multicolumn{6}{|l|}{ Males } \\
\hline \multirow[t]{2}{*}{ Akune et $\mathrm{al}^{29}$} & 349 & Current walking habits & Yes vs no & Age and BMI & $0.60(0.28-1.27)$ \\
\hline & & Exercise habits in middle age & Yes vs no & Age and $\mathrm{BMI}$ & $0.48(0.22-1.03)$ \\
\hline Castillo et al ${ }^{62}$ & 694 & Exercise 3+ times/wk & Yes vs no & $\begin{array}{l}\text { Age, alcohol use, and current } \\
\text { smoking status }\end{array}$ & $0.77(0.39-1.55)$ \\
\hline
\end{tabular}


Table 3 (Continued)

\begin{tabular}{|c|c|c|c|c|c|}
\hline Study & $\mathbf{N}$ & Variable & Status & $\begin{array}{l}\text { Multiple logistic regression models } \\
\text { adjusted for }\end{array}$ & OR $(95 \% \mathrm{Cl})$ \\
\hline $\begin{array}{l}\text { Figueiredo } \\
\text { et } \text { al }^{64}\end{array}$ & 399 & Physical activity & Yes vs no & $\begin{array}{l}\text { Age, BMI, race, smoking, and total femur } \\
\text { bone mineral density }\end{array}$ & $0.28(0.08-0.95)^{*}$ \\
\hline \multirow[t]{2}{*}{$\begin{array}{l}\text { Goodman } \\
\text { et } \mathrm{al}^{49}\end{array}$} & 551 & $\begin{array}{l}\text { Average level of physical } \\
\text { activity each day }\end{array}$ & Moderate vs low & Unadjusted & $0.79(0.62-1.01)$ \\
\hline & & & Heavy vs low & Unadjusted & $0.57(0.29-1.13)$ \\
\hline \multirow[t]{5}{*}{ Kim et $\mathrm{al}^{30}$} & $\mathrm{I}, 156$ & $\begin{array}{l}3 \text { or more days of vigorous } \\
\text { activity of at least } 20 \mathrm{~min} \\
\text { per day }\end{array}$ & - & $\begin{array}{l}\text { Age, BMI, smoking habit, alcohol } \\
\text { drinking, family income, education, and } \\
\text { protein intake }\end{array}$ & $0.55(0.23-1.31)$ \\
\hline & & $\begin{array}{l}5 \text { or more days of moderate- } \\
\text { intensity activity of at least } \\
30 \mathrm{~min} / \mathrm{d}\end{array}$ & - & $\begin{array}{l}\text { Age, BMI, smoking habit, alcohol } \\
\text { drinking, family income, education, and } \\
\text { protein intake }\end{array}$ & $0.59(0.26-1.36)$ \\
\hline & & $\begin{array}{l}5 \text { or more days of walking of } \\
\text { at least } 30 \mathrm{~min} / \mathrm{d}\end{array}$ & - & $\begin{array}{l}\text { Age, BMI, smoking habit, alcohol } \\
\text { drinking, family income, education, and } \\
\text { protein intake }\end{array}$ & $0.49(0.29-0.83)^{*}$ \\
\hline & & $\begin{array}{l}\text { Strength exercise: } 2 \text { or more } \\
\text { days/wk }\end{array}$ & - & $\begin{array}{l}\text { Age, BMI, smoking habit, alcohol } \\
\text { drinking, family income, education and } \\
\text { protein intake }\end{array}$ & $0.59(0.24-\mathrm{I} .48)$ \\
\hline & & $\begin{array}{l}\text { Flexibility exercise: } 2 \text { or more } \\
\text { days/wk }\end{array}$ & - & $\begin{array}{l}\text { Age, BMI, smoking habit, alcohol } \\
\text { drinking, family income, education and } \\
\text { protein intake }\end{array}$ & I.2I $(0.6 \mathrm{I}-2.40)$ \\
\hline \multirow[t]{2}{*}{ Ryu et $\mathrm{al}^{3 !}$} & 940 & Physical activity level & Moderate vs low & Age & $0.65(0.4 I-1.04)$ \\
\hline & & & High vs low & Age & $0.29(0.15-0.56)^{*}$ \\
\hline \multicolumn{6}{|c|}{ Females and males together } \\
\hline \multirow[t]{2}{*}{ Akune et $\mathrm{al}^{29}$} & 1,000 & Current walking habits & Yes vs no & Age and BMI & $0.69(0.42-1.12)$ \\
\hline & & Exercise habits in middle age & Yes vs no & Age and $\mathrm{BMI}$ & $0.53(0.31-0.90)^{*}$ \\
\hline \multirow[t]{3}{*}{ Beavers et $\mathrm{al}^{45}$} & 7,544 & Physical activity level & High vs none & Age, BMI, protein intake, serum uric acid & $0.80(0.70-1.00)^{*}$ \\
\hline & & & Med vs none & Age, BMI, protein intake, serum uric acid & $0.70(0.60-1.00)^{*}$ \\
\hline & & & Low vs none & Age, BMI, protein intake, serum uric acid & $0.80(0.60-1.00) *$ \\
\hline da Silva et $\mathrm{al}^{47}$ & 253 & Past physical activity (PPA) & Yes vs no & Unclear & $0.4 I(0.20-0.82)^{*}$ \\
\hline Landi et $\mathrm{al}^{50}$ & 122 & I $\mathrm{h}$ or more exercises per day & Never or less than I h/d & Unadjusted & $0.37(0.12-0.99)^{*}$ \\
\hline \multirow[t]{2}{*}{ Murphy et al ${ }^{54}$} & 2,355 & Physical activity & $500-\mathrm{l}, 499$ vs $<500 \mathrm{kcal} / \mathrm{wk}$ & Unclear & $0.87(0.70-1.06)$ \\
\hline & & & $>\mathrm{I}, 500 \mathrm{vs}<500 \mathrm{kcal} / \mathrm{wk}$ & Unclear & $0.77(0.60-0.97)^{*}$ \\
\hline Yo et $\mathrm{al}^{66}$ & 3,142 & PASE total score & - & $\begin{array}{l}\text { Age, demographics, socioeconomic status, } \\
\text { medical history, lifestyle factors, cognitive } \\
\text { function, IADL impairments, and BMI }\end{array}$ & $0.99(0.98-0.99)^{*}$ \\
\hline Zeng et $\mathrm{al}^{59, \mathrm{a}}$ & $46 I$ & Physical exercise & Yes vs no & Unclear & $0.27(0.09-0.79)^{*}$ \\
\hline
\end{tabular}

Notes: aSarcopenia was considered as low gait speed. *Statistically significant.

Abbreviations: OR, odds ratio; Cl, confidence interval; BMI, body mass index; PASE, Physical Activity Scale of the Elderly; LDL, low-density lipoproteins; HDL, high-density lipoproteins; WHR, waist-hip relation; WC, waist circumference; $\mathrm{Cl}$, conicity index; WHER, waist-height relation; IADL, instrumental activity of daily living.

Table 4 Relationship between sarcopenia and physical inactivity, according to multiple regression models

\begin{tabular}{|c|c|c|c|c|c|c|}
\hline Study & Sex & $\mathbf{N}$ & Variable & $\begin{array}{l}\text { Multiple logistic } \\
\text { regression models } \\
\text { adjusted for }\end{array}$ & Status & OR (95\% CI) \\
\hline Atkins et $\mathrm{al}^{61}$ & Males & 4,252 & Physically inactive & Age & - & $1.43(1.15-1.76)^{*}$ \\
\hline Dutra et al $^{48}$ & Females & 173 & Sedentary & Crude & vs active & $2.96(1.23-7.12)^{*}$ \\
\hline \multirow[t]{2}{*}{ Lau et $\mathrm{al}^{51}$} & Males & 262 & Regular exercise & Age & No vs yes & I.5I (0.68-3.38) \\
\hline & Females & 265 & Regular exercise & Age & No vs yes & $1.10(0.40-3.00)$ \\
\hline \multirow[t]{2}{*}{ Lin et $\mathrm{al}^{52}$} & Together & 761 & Exercise & Crude & No vs yes & $3.09(1.98-4.82)^{*}$ \\
\hline & & & Exercise & $\begin{array}{l}\text { Age, sex, marital } \\
\text { status, regular } \\
\text { exercise habits, } \\
\text { comorbidity status }\end{array}$ & No vs yes & $2.70(I .66-4.4 I)^{*}$ \\
\hline Martinez et $\mathrm{al}^{53}$ & Together & 110 & $\begin{array}{l}\text { Physical activity less } \\
\text { than } 2 \times \text { per week }\end{array}$ & Unclear & & $3.40(1.10-10.90)^{*}$ \\
\hline $\begin{array}{l}\text { Silva Alexandre } \\
\text { et } \mathrm{al}^{56}\end{array}$ & Together & $\mathrm{I}, 149$ & Sedentary lifestyle & Unclear & vs active lifestyle & $0.66(0.42-1.06)$ \\
\hline Tramontano et $\mathrm{al}^{57}$ & Together & 222 & $\begin{array}{l}\text { Low physical } \\
\text { activity levels }\end{array}$ & Unclear & $\begin{array}{l}\text { Recommended } \\
\text { physical activity levels }\end{array}$ & $3.80(1.30-10.90)^{*}$ \\
\hline
\end{tabular}

Note: *Statistically significant.

Abbreviations: $\mathrm{OR}$, odds ratio; $\mathrm{Cl}$, confidence interval. 


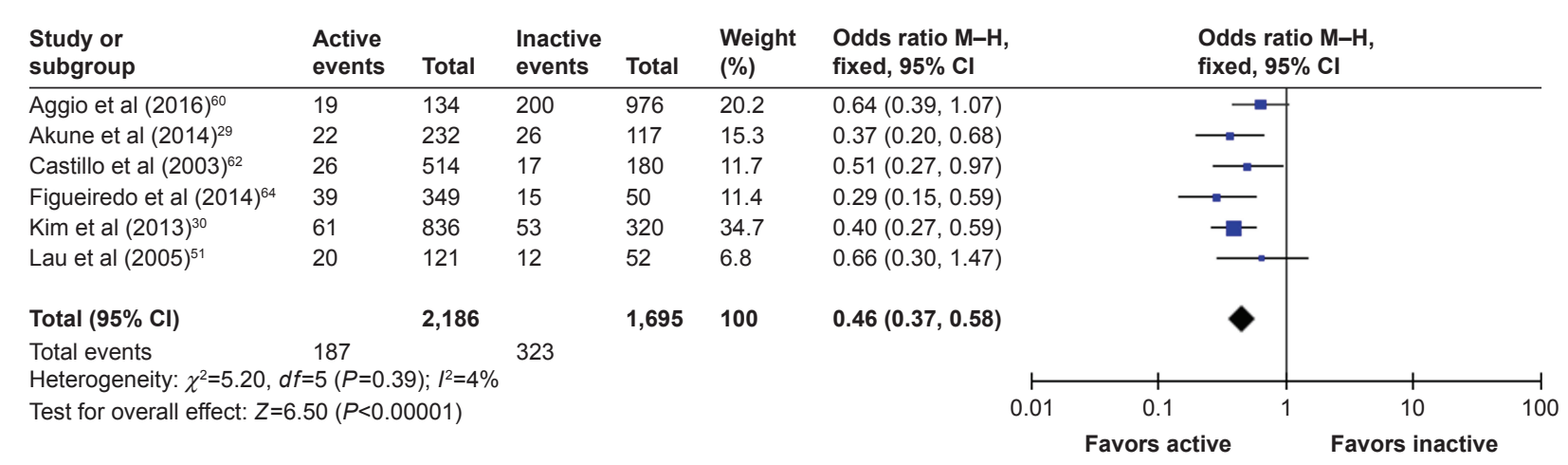

Figure 2 The forest plot of effect sizes for males. Abbreviation: $\mathrm{Cl}$, confidence interval.

three recent meta-analyses: one including eight trials reporting that exercise can increase gait speed, balance, and activities of daily living in frail older adults, ${ }^{67}$ another incorporating 19 trials that concluded that exercise has some benefits in frail older people ${ }^{68}$ and a third synthesizing data from 18 studies, which provided evidence that physical exercise therapy could improve mobility and physical functioning even among older patients with mobility problems and physical disability. ${ }^{69}$ Although there is conformity among our work and these meta-analyses, it should be pointed out that the other metaanalyses were focused mostly on randomized controlled trials while our meta-analysis combined diverse studies of PA, which was typically identified by self-report. It is worth mentioning that the method of acquiring PA data largely varies among studies. In involved studies, some people performed PA such as regular housework, gardening, or did an occupational activity involving the carrying of light or heavy objects. They also occasionally walked, did slow swimming, played doubles tennis, volleyball, did vigorous exercise such as running, climbing, fast cycling, fast swimming, football, basketball, rope jumping, squash, and singles tennis. In the study of Aggio et al, ${ }^{60}$ participants wore an accelerometer for 7 days during waking hours, which was removed only for water-based activities.

As seen in Table 2, many different methods were used to diagnose sarcopenia, which may result in increased risk of publication bias, which has been previously described. ${ }^{70}$ For example, Goodman et $\mathrm{al}^{49}$ used only one standard deviation below a young reference group as the cutoff value for diagnosing sarcopenia, which may have caused a large percentage of the population to be falsely identified as sarcopenic. Another weakness of our review was that we did not include subgroup analyses, as there were only a few studies for making subgroups according to sarcopenia diagnosing or several different physical activities as well as metabolic equivalent of task (MET). Therefore, we recommend that future research should unify diagnostic methods according to consensus. This may improve our knowledge of how PA plays a role in sarcopenia protection. Finally, it should be mentioned that we used only four databases and the terms "sarcopenia" and "physical activity" may not have unearthed $100 \%$ of the research in this area. However, we believe that despite this limitation, the review is beneficial, as it is the first systematic review and meta-analysis on the topic.

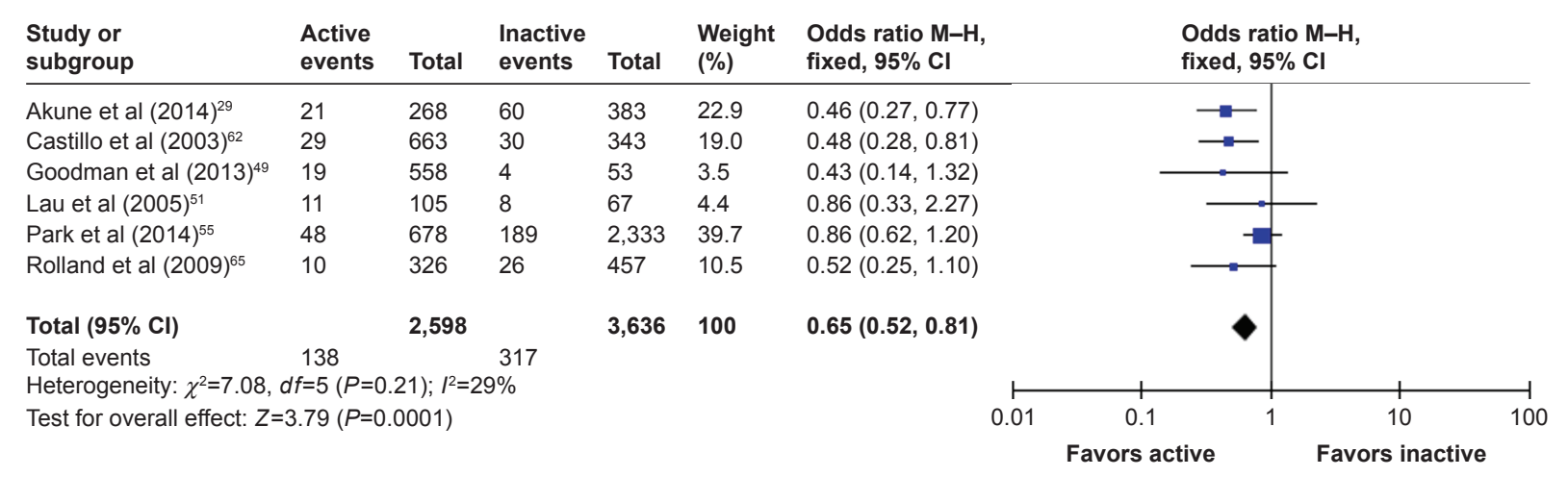

Figure 3 The forest plot of effect sizes for females. 


\begin{tabular}{|c|c|c|c|}
\hline $\begin{array}{l}\text { Study or } \\
\text { subgroup }\end{array}$ & $\begin{array}{l}\text { Active } \\
\text { events }\end{array}$ & Total & $\begin{array}{l}\text { Inactiv } \\
\text { events }\end{array}$ \\
\hline Akune et al $(2014)^{29}$ & 43 & 500 & 86 \\
\hline Castillo et al $(2003)^{62}$ & 55 & 1,177 & 47 \\
\hline Landi et al $(2012)^{50}$ & 20 & 84 & 20 \\
\hline Lau et al $(2005)^{51}$ & 31 & 226 & 20 \\
\hline Lin et al $(2013)^{52}$ & 55 & 583 & 44 \\
\hline Martinez et al $(2015)^{53}$ & 4 & 34 & 19 \\
\hline Volpato et al (2014) $)^{58}$ & 7 & 128 & 48 \\
\hline Total $(95 \% \mathrm{Cl})$ & & 2,732 & \\
\hline Total events & 215 & & 284 \\
\hline
\end{tabular}

Figure 4 The forest plot of effect sizes for males and females.

In summary, when participants did at least some PA, they had better odds of avoiding sarcopenia. Our results support the recommendation of the American College of Sports Medicine (ACSM) and the American Heart Association (AHA) that regular PA, including occupational activity, aerobic sport activity, and muscle-strengthening activity, is essential for healthy aging. ${ }^{71}$

Most likely, the association between PA and the protection of muscle mass is common sense. However, this is the first systematic review and meta-analysis to confirm this association on the basis of cross-sectional and cohort studies. Moreover, it seems that the type of PA that is undertaken is not important, because except for one study that showed an association between PA and worsening sarcopenia, studies including several different PA showed that PA acts as a protective factor against sarcopenia.

\section{Acknowledgments}

The research presented in this article was carried out as part of the Marie Curie Initial Training Network (ITN) action, H2020-MSCA-ITN-2015, under grant agreement number 676265, the AZV research projects 16-29182A, and 15-32942A-P09 of the Ministry of Health and projects Q 41 and P 20.

\section{Disclosure}

The authors report no conflicts of interest in this work.

\section{References}

1. Nelson ME, Rejeski WJ, Blair SN, et al. Physical activity and public health in older adults: recommendation from the American College of Sports Medicine and the American Heart Association. Med Sci Sports Exerc. 2007;39(8):1435-1445.

2. Fletcher GF, Balady GJ, Amsterdam EA, et al. Exercise standards for testing and training: a statement for healthcare professionals from the American Heart Association. Circulation. 2001;104(14):1694-1740.
3. Force USPST. Screening for obesity in adults: recommendations and rationale. Ann Intern Med. 2003;139(11):930-932.

4. Sigal RJ, Kenny GP, Wasserman DH, Castaneda-Sceppa C. Physical activity/exercise and type 2 diabetes. Diabetes Care. 2004;27(10): 2518-2539.

5. Chobanian AV, Bakris GL, Black HR, et al. The seventh report of the Joint National Committee on prevention, detection, evaluation, and treatment of high blood pressure: the JNC 7 report. JAMA. 2003;289(19): 2560-2572.

6. McDermott MM, Liu K, Ferrucci L, et al. Physical performance in peripheral arterial disease: a slower rate of decline in patients who walk more. Ann Intern Med. 2006;144(1):10-20.

7. Geliebter A, Maher MM, Gerace L, Gutin B, Heymsfield SB, Hashim SA. Effects of strength or aerobic training on body composition, resting metabolic rate, and peak oxygen consumption in obese dieting subjects. Am J Clin Nutr. 1997;66(3):557-563.

8. Going S, Lohman T, Houtkooper L, et al. Effects of exercise on bone mineral density in calcium-replete postmenopausal women with and without hormone replacement therapy. Osteoporos Int. 2003;14(8): 637-643.

9. American Geriatrics Society Panel on Exercise and Osteoarthritis. Exercise prescription for older adults with osteoarthritis pain: consensus practice recommendations. A supplement to the AGS Clinical Practice Guidelines on the management of chronic pain in older adults. $J$ Am Geriatr Soc. 2001;49(6):808-823.

10. Pauwels RA, Buist AS, Calverley PM, Jenkins CR, Hurd SS, Committee GS. Global strategy for the diagnosis, management, and prevention of chronic obstructive pulmonary disease. NHLBI/WHO Global Initiative for Chronic Obstructive Lung Disease (GOLD) Workshop summary. Am J Respir Crit Care Med. 2001;163(5):1256-1276.

11. Breen L, Phillips SM. Interactions between exercise and nutrition to prevent muscle waste during ageing. Br J Clin Pharmacol. 2013; 75(3):708-715.

12. Rosenberg IH. Epidemiologic and methodologic problems in determining nutritional-status of older persons - proceedings of a conference held in Albuquerque, New Mexico, October 19-21, 1988 - summary comments. Am J Clin Nutr. 1989;50(5):1231-1233.

13. Aoyagi Y, Shephard RJ. Sex differences in relationships between habitual physical activity and health in the elderly: practical implications for epidemiologists based on pedometer/accelerometer data from the Nakanojo Study. Arch Gerontol Geriatr. 2013;56(2):327-338.

14. Di Tano G, Fulle S, Pietrangelo T, Bellomo R, Fanò G. Sarcopenia: characteristics, genesis, remedies. Sport Sci Health. 2005;1(2):69-74.

15. FreibergerE, Sieber C, Pfeifer K. Physical activity, exercise, and sarcopeniafuture challenges. Wien Med Wochenschr. 2011;161(17-18):416-425.

16. Baumgartner RN, Koehler KM, Gallagher D, et al. Epidemiology of sarcopenia among the Elderly in New Mexico. Am J Epidemiol. 1998; 147(8):755-763. 
17. Cruz-Jentoft AJ, Baeyens JP, Bauer JM, et al. Sarcopenia: European consensus on definition and diagnosis: report of the European Working Group on sarcopenia in older people. Age Ageing. 2010;39(4): $412-423$.

18. Janssen I, Heymsfield SB, Baumgartner RN, Ross R. Estimation of skeletal muscle mass by bioelectrical impedance analysis. $J$ Appl Physiol. 2000;89(2):465-471.

19. Landi F, Russo A, Liperoti R, et al. Midarm muscle circumference, physical performance and mortality: results from the aging and longevity study in the Sirente geographic area (ilSIRENTE study). Clin Nutr. 2010;29(4):441-447.

20. Chen LK, Liu LK, Woo J, et al. Sarcopenia in Asia: consensus report of the Asian Working Group for sarcopenia. J Am Med Dir Assoc. 2014; 15(2):95-101.

21. Fielding RA, Vellas B, Evans WJ, et al. Sarcopenia: an undiagnosed condition in older adults. Current consensus definition: prevalence, etiology, and consequences. International working group on sarcopenia. J Am Med Dir Assoc. 2011;12(4):249-256.

22. Buford TW, Anton SD, Judge AR, et al. Models of accelerated sarcopenia: critical pieces for solving the puzzle of age-related muscle atrophy. Ageing Res Rev. 2010;9(4):369-383.

23. Evans WJ. Skeletal muscle loss: cachexia, sarcopenia, and inactivity. Am J Clin Nutr. 2010;91(4):1123S-1127S.

24. Petrella JK, Kim JS, Tuggle SC, Bamman MM. Contributions of force and velocity to improved power with progressive resistance training in young and older adults. Eur J Appl Physiol. 2007;99(4):343-351.

25. Granacher U, Lacroix A, Muehlbauer T, Roettger K, Gollhofer A. Effects of core instability strength training on trunk muscle strength, spinal mobility, dynamic balance and functional mobility in older adults. Gerontology. 2013;59(2):105-113.

26. Candow DG, Chilibeck PD, Abeysekara S, Zello GA. Short-term heavy resistance training eliminates age-related deficits in muscle mass and strength in healthy older males. J Strength Cond Res. 2011;25(2): 326-333.

27. Kosek DJ, Kim JS, Petrella JK, Cross JM, Bamman MM. Efficacy of 3 days/wk resistance training on myofiber hypertrophy and myogenic mechanisms in young vs. older adults. J Appl Physiol. 2006;101(2): 531-544.

28. Park H, Park S, Shephard RJ, Aoyagi Y. Yearlong physical activity and sarcopenia in older adults: the Nakanojo Study. Eur J Appl Physiol. 2010; 109(5):953-961.

29. Akune T, Muraki S, Oka H, et al. Exercise habits during middle age are associated with lower prevalence of sarcopenia: the ROAD study. Osteoporos Int. 2014;25(3):1081-1088.

30. Kim SH, Kim TH, Hwang HJ. The relationship of physical activity (PA) and walking with sarcopenia in Korean males aged 60 years and older using the Fourth Korean National Health and Nutrition Examination Survey (KNHANES IV-2, 3), 2008-2009. Arch Gerontol Geriatr. 2013;56(3):472-477.

31. Ryu M, Jo J, Lee Y, Chung YS, Kim KM, Baek WC. Association of physical activity with sarcopenia and sarcopenic obesity in communitydwelling older adults: the Fourth Korea National Health and Nutrition Examination Survey. Age Ageing. 2013;42(6):734-740.

32. Aagaard P, Suetta C, Caserotti P, Magnusson SP, Kjaer M. Role of the nervous system in sarcopenia and muscle atrophy with aging: strength training as a countermeasure. Scand J Med Sci Sports. 2010;20(1): 49-64.

33. Burton LA, Sumukadas D. Optimal management of sarcopenia. Clin Interv Aging. 2010;5:217-228.

34. Candow DG. Sarcopenia: current theories and the potential beneficial effect of creatine application strategies. Biogerontology. 2011;12(4): 273-281.

35. Forbes SC, Little JP, Candow DG. Exercise and nutritional interventions for improving aging muscle health. Endocrine. 2012;42(1):29-38.

36. Hollmann W, Strüder HK, Tagarakis CVM, King G. Physical activity and the elderly. Eur J Cardiovasc Prev Rehabil. 2007;14(6):730-739.

37. Kamel HK. Sarcopenia and aging. Nutr Rev. 2003;61(5 pt 1):157-167.
38. Mayer F, Scharhag-Rosenberger F, Carlsohn A, Cassel M, Muller S, Scharhag J. The intensity and effects of strength training in the elderly. Dtsch Arztebl Int. 2011;108(21):359-364.

39. Liberati A, Altman DG, Tetzlaff J, et al. The PRISMA statement for reporting systematic reviews and meta-analyses of studies that evaluate healthcare interventions: explanation and elaboration. $B M J$. 2009;339:b2700.

40. Wells GA, Shea B, O'Connell D, et al [webpage on the Internet]. The Newcastle-Ottawa Scale (NOS) for Assessing the Quality of Nonrandomised Studies in Meta-Analyses. Available from: http://www.ohri. ca/programs/clinical_epidemiology/oxford.htm. Accessed March 27, 2017.

41. Mantel N, Haenszel W. Statistical aspects of the analysis of data from retrospective studies of disease. J Natl Cancer Inst. 1959;22(4):719-748.

42. Higgins JP, Thompson SG, Deeks JJ, Altman DG. Measuring inconsistency in meta-analyses. BMJ. 2003;327(7414):557-560.

43. Egger M, Davey Smith G, Schneider M, Minder C. Bias in meta-analysis detected by a simple, graphical test. BMJ. 1997;315(7109):629-634.

44. Higgins J, Green S. Cochrane handbook for systematic reviews of interventions version 5.1.0. In: Higgins J, Green S, editors. The Cochrane Collaboration. 2011. Available from: http://handbook.cochrane.org/

45. Beavers KM, Beavers DP, Serra MC, Bowden RG, Wilson RL. Low relative skeletal muscle mass indicative of sarcopenia is associated with elevations in serum uric acid levels: findings from NHANES III. J Nutr Health Aging. 2009;13(3):177-182.

46. de Castro EA, Lima LM, Cerqueira MS, Gobbi S, Doimo LA. Sarcopenia and cardiovascular risk in physically active adult and elderly women. Motriz J Phys Ed. 2014;20:92-99.

47. da Silva AP, Matos A, Ribeiro R, et al. Sarcopenia and osteoporosis in Portuguese centenarians. Eur J Clin Nutr. 2017;71(1):56-63.

48. Dutra T, Pinheiro PA, Carneiro JAO, Coqueiro RDS, Fernandes MH. Prevalence and factors associated with sarcopenia in elderly women living in the community. Rev Bras Cineantropom Desempenho Hum. 2015;17:460-471.

49. Goodman MJ, Ghate SR, Mavros P, et al. Development of a practical screening tool to predict low muscle mass using NHANES 1999-2004. J Cachexia Sarcopenia Muscle. 2013;4(3):187-197.

50. Landi F, Liperoti R, Fusco D, et al. Prevalence and risk factors of sarcopenia among nursing home older residents. J Gerontol A Biol Sci Med Sci. 2012;67(1):48-55.

51. Lau EM, Lynn HS, Woo JW, Kwok TC, Melton LJ 3rd. Prevalence of and risk factors for sarcopenia in elderly Chinese men and women. J Gerontol A Biol Sci Med Sci. 2005;60(2):213-216.

52. Lin CC, Lin WY, Meng NH, et al. Sarcopenia prevalence and associated factors in an elderly Taiwanese metropolitan population. J Am Geriatr Soc. 2013;61(3):459-462.

53. Martinez BP, Batista AK, Gomes IB, Olivieri FM, Camelier FW, Camelier AA. Frequency of sarcopenia and associated factors among hospitalized elderly patients. BMC Musculoskelet Disord. 2015; 16:108.

54. Murphy RA, Ip EH, Zhang Q, et al. Transition to sarcopenia and determinants of transitions in older adults: a population-based study. J Gerontol A Biol Sci Med Sci. 2014;69(6):751-758.

55. Park S, Ham JO, Lee BK. A positive association of vitamin D deficiency and sarcopenia in 50 year old women, but not men. Clin Nutr. 2014;33(5):900-905.

56. Silva Alexandre T, Oliveira Duarte YA, Santos JLF, Wong R, Lebrão ML. Prevalence and associated factors of sarcopenia among elderly in Brazil: findings from the SABE study. J Nutr Health Aging. 2014;18(3): 284-290.

57. Tramontano A, Veronese N, Sergi G, et al. Prevalence of sarcopenia and associated factors in the healthy older adults of the Peruvian Andes. Arch Gerontol Geriatr. 2017;68:49-54.

58. Volpato S, Bianchi L, Cherubini A, et al. Prevalence and clinical correlates of sarcopenia in community-dwelling older people: application of the EWGSOP definition and diagnostic algorithm. J Gerontol A Biol Sci Med Sci. 2014;69(4):438-446. 
59. Zeng P, Han Y, Pang J, et al. Sarcopenia-related features and factors associated with lower muscle strength and physical performance in older Chinese: a cross sectional study. BMC Geriatr. 2016;16:45.

60. Aggio DA, Sartini C, Papacosta O, et al. Cross-sectional associations of objectively measured physical activity and sedentary time with sarcopenia and sarcopenic obesity in older men. Prev Med. 2016;91: 264-272.

61. Atkins JL, Whincup PH, Morris RW, Wannamethee SG. Low muscle mass in older men: the role of lifestyle, diet and cardiovascular risk factors. J Nutr Health Aging. 2014;18(1):26-33.

62. Castillo EM, Goodman-Gruen D, Kritz-Silverstein D, Morton DJ, Wingard DL, Barrett-Connor E. Sarcopenia in elderly men and women: the Rancho Bernardo study. Am J Prev Med. 2003;25(3):226-231.

63. Domiciano DS, Figueiredo CP, Lopes JB, et al. Discriminating sarcopenia in community-dwelling older women with high frequency of overweight/obesity: the Sao Paulo Ageing \& Health Study (SPAH). Osteoporos Int. 2013;24(2):595-603.

64. Figueiredo CP, Domiciano DS, Lopes JB, et al. Prevalence of sarcopenia and associated risk factors by two diagnostic criteria in communitydwelling older men: the Sao Paulo Ageing \& Health Study (SPAH). Osteoporos Int. 2014;25(2):589-596.

65. Rolland Y, Lauwers-Cances V, Cristini C, et al. Difficulties with physical function associated with obesity, sarcopenia, and sarcopenic-obesity in community-dwelling elderly women: the EPIDOS (EPIDemiologie de l'OSteoporose) Study. Am J Clin Nutr. 2009;89(6):1895-1900.

66. Yu R, Wong M, Leung J, Lee J, Auyeung TW, Woo J. Incidence, reversibility, risk factors and the protective effect of high body mass index against sarcopenia in community-dwelling older Chinese adults. Geriatr Gerontol Int. 2014;14(suppl 1):15-28.

67. Chou CH, Hwang CL, Wu YT. Effect of exercise on physical function, daily living activities, and quality of life in the frail older adults: a meta-analysis. Arch Phys Med Rehabil. 2012;93(2):237-244.

68. Gine-Garriga M, Roque-Figuls M, Coll-Planas L, Sitja-Rabert M, Salva A. Physical exercise interventions for improving performancebased measures of physical function in community-dwelling, frail older adults: a systematic review and meta-analysis. Arch Phys Med Rehabil. 2014;95(4):753-769.e3.

69. de Vries NM, van Ravensberg CD, Hobbelen JSM, Olde Rikkert MGM, Staal JB, Nijhuis-van der Sanden MWG. Effects of physical exercise therapy on mobility, physical functioning, physical activity and quality of life in community-dwelling older adults with impaired mobility, physical disability and/or multi-morbidity: a meta-analysis. Ageing Res Rev. 2012;11(1):136-149.

70. Steffl M, Bohannon R, Petr M, Kohlikova E, Holmerova I. Alcohol consumption as a risk factor for sarcopenia - a meta-analysis. BMC Geriatr. 2016;16(1):99.
71. Nelson ME, Rejeski WJ, Blair SN, et al. Physical activity and public health in older adults: recommendation from the American College of Sports Medicine and the American Heart Association. Circulation. 2007; 116(9):1094-1105.

72. Miller MD, Crotty M, Giles LC, et al. Corrected arm muscle area: an independent predictor of long-term mortality in community-dwelling older adults? J Am Geriatr Soc. 2002;50(7):1272-1277.

73. Pichard C, Kyle UG, Bracco D, Slosman DO, Morabia A, Schutz Y. Reference values of fat-free and fat masses by bioelectrical impedance analysis in 3393 healthy subjects. Nutrition. 2000;16(4):245-254.

74. Matsudo SM, Araújo T, Matsudo VKR, et al. International physical activity questionnaire (IPAQ): study of validity and reliability in Brazil. Rev Bras Ativ Fís Saúde. 2001;6(2):5-18.

75. Atkins JL, Whincup PH, Morris RW, Lennon LT, Papacosta O, Wannamethee SG. Sarcopenic obesity and risk of cardiovascular disease and mortality: a population-based cohort study of older men. J Am Geriatr Soc. 2014;62(2):253-260.

76. Fitti JE, Kovar MG. The supplement on aging to the 1984 National Health Interview Survey. Vital Health Stat 1. 1987;(21):1-115.

77. Craig CL, Marshall AL, Sjostrom M, et al. International physical activity questionnaire: 12-country reliability and validity. Med Sci Sports Exerc. 2003;35(8):1381-1395.

78. Lee RC, Wang Z, Heo M, Ross R, Janssen I, Heymsfield SB. Total-body skeletal muscle mass: development and cross-validation of anthropometric prediction models. Am J Clin Nutr. 2000;72(3):796-803.

79. Janssen I, Baumgartner RN, Ross R, Rosenberg IH, Roubenoff R. Skeletal muscle cutpoints associated with elevated physical disability risk in older men and women. Am J Epidemiol. 2004;159(4):413-421.

80. Bernabei R, Landi F, Onder G, Liperoti R, Gambassi G. Second and third generation assessment instruments: the birth of standardization in geriatric care. J Gerontol A Biol Sci Med Sci. 2008;63(3):308-313.

81. Morris J, Hawes C, Murphy K, et al. Minimum Data Set Plus Training Manual. Natick, MA: Eliot Press; 1991

82. Gallagher D, Visser M, De Meersman RE, et al. Appendicular skeletal muscle mass: effects of age, gender, and ethnicity. J Appl Physiol (1985). 1997;83(1):229-239.

83. Armstrong T, Bull F. Development of the World Health Organization Global Physical Activity Questionnaire (GPAQ). J Public Health. 2006; 14(2):66-70.

84. Washburn RA, Smith KW, Jette AM, Janney CA. The Physical Activity Scale for the Elderly (PASE): development and evaluation. J Clin Epidemiol. 1993;46(2):153-162.
Clinical Interventions in Aging

\section{Publish your work in this journal}

Clinical Interventions in Aging is an international, peer-reviewed journal focusing on evidence-based reports on the value or lack thereof of treatments intended to prevent or delay the onset of maladaptive correlates of aging in human beings. This journal is indexed on PubMed Central, MedLine,

\section{Dovepress}

CAS, Scopus and the Elsevier Bibliographic databases. The manuscript management system is completely online and includes a very quick and fair peer-review system, which is all easy to use. Visit http://www.dovepress. com/testimonials.php to read real quotes from published authors. 